not stand still ; it would either expand or contract. To avoid this apparent absurdity, Einstein imagined the continuum endowed with a further curvature of its own, independent of the presence of matter and so inherent in the space itself. This was specified by a quantity, the 'cosmical constant', which was supposed to have a uniform value everywhere and so kept the total volume of space fixed and unalterable.

There is no observational evidence that such a constant exists, for the curvature it implies is too small for measurement. The constant was only introduced because Einstein had thought space must be at rest, and there is no need to retain it now that space appears not to be at rest. On the other hand, we are under no compulsion to discard it. Actually Einstein and de Sitter have found that the constant can have a large range of values, including zero, without running counter to any of the observed facts of astronomy.

We may compare space-time to a river having space as its cross-section and time as the direction of flow of its stream. Two dimensions are, of course, missing; the cross-section of our river ought to have three dimensions instead of one, but as all three are all exactly similar, the suppression of two of them does no great harm.

If space could remain constant in size, this river would become a canal with parallel banks; Einstein's original space-time river was of this type. But Friedmann and Lemaître showed that such a space would be unstable; any slight disturbance or irregularity-such as would, for instance, be caused by the condensation of a primeval gas into nebulæ-would start it either expanding or contracting. For this reason Lemaître thought that the Einstein canal should be replaced by a sort of Amazon River, starting from minute beginnings and for ever widening as it flows-expanding space. De Sitter found that other values for the cosmical constant made two other types of solution mathematically possible. In one of these the canal-like river gives place to a sort of Panama Canal - space first contracts until it reaches a minimum and then expands again to an indefinite extent. In the other, space rhythmically expands and contracts, so that the spacetime river becomes a series of regularly spaced lakes connected by narrows.

The Amazon-like space-time river of Lemaître was open to one grave objection. Its length, which is time-the whole time since the beginning of the universe-was limited, and its source was nothing like distant enough to allow for the observed stages of development of stellar systems -in brief, the stars were too old to have grown up within the length of the river.

The two more recent solutions of de Sitter and Einstein are not open to any such objection, and at present either of them appears capable of providing a true, although highly artificial, representation of the observed phenomena of the universe. At one time, de Sitter was advocating the Panama canal type of map, while Einstein favoured the rhythmical universe of lake and narrows-a space which alternately expanded and contracted. Einstein now appears to contemplate the possibility of a zero cosmical constant and a space of infinite extent. But it is, I think, fair to say that no one is satisfied with the present position. It may be that still other alternatives remain to be discovered, and another few years may witness some new formulation of the problem which will lead to a satisfactory solution.

\title{
The New Age in Physics
}

\section{By Dr. H. Dingle, Imperial College of Science and Technology, London}

$\mathrm{E}^{\mathrm{n}}$ VERY advance in thought has two aspects - the loss of the old and the gain of the new-and it is probably inevitable that, after the first flush of excitement has faded away, the former should become the more conspicuous. It may inspire joy at the passing of a delusion, or regret at the failure of an ideal : in either case it is the negative aspect of the change which protrudes itself, because all are conscious that what they believed in has gone, but only a few can at first see the significance of the new thing which has come.

This is exemplified by the fact, which is in all our minds to-day, that King George V has occupied the throne of England for twenty-five years. What does it mean? In 1910 we knew well enough what it would mean; but in 1935, who except a mathematical physicist will commit himself to an opinion? Twenty-five years to one observer, we are told, may be fifty years to another, and neither can claim superiority for his time-scale. Why, then, not celebrate a golden instead of a silver jubilee? The relativist knows, of course, that the destruction of absolute time is merely the necessary preliminary to the building of an absolute 'interval', and that twenty-five years is the interval during which King George has reigned. In this matter His Majesty's time is proper time, so that physics 
and patriotism support one another. But for one to whom this is the significant aspect of relativity, there are a thousand who know only that where they thought was certainty there is only confusion.

It will not be amiss, therefore, to look at the positive side of the changes in physical thought which the past twenty-five years have seen. What principles have been introduced into science during that time, or having already existed there, have been more clearly understood and more rigidly applied? How does this epoch appear against the broad background of scientific history? What is the character of the tide as distinct from the wanderings of individual waves? Only future historians can give final answers to these questions, but we may attempt to answer them in a manner fitted to the needs of our time.

There seems little doubt that the essential contribution of relativity to science is the principle usually known as 'the rejection of unobservables'. It is not new in the sense that it has never before been applied: on the contrary, it is exemplified in almost every forward movement which physics has made. But it has been used unconsciously, instinctively, and therefore to some extent inconsistently. It has now been brought into the light of day. That is the significant thing, beside which the consequences to our understanding of mechanics and gravitation are of secondary importance.

It is very doubtful if this principle has yet been properly formulated, and quite certain that its full implications have not yet been grasped. It is probable, too, that it has on occasion been wrongly used. Though in appearance a merely negative principle, it is in fact a positive instrument of incalculable power. We venture to suggest the following two statements as a provisional expression of its meaning :

(1) The criterion of objective physical existence is general observability by physical means*.

(2) In the logical correlation of experience, the concepts employed shall be such that whatever is not generally observable by physical means is necessarily meaningless.

Particular attention should be directed to the word 'necessarily'. It is not enough to reject unobservables : we must frame our laws of Nature so that they cannot arise to be rejected. It is this that makes the principle an inherently positive one. Its first fully conscious application by Einstein illustrates this excellently. It is not sufficient merely to say that because it is impossible to observe motion relative to the ether, such motion is meaningless. We must define the

* That is, intrinsic observahility ; for example, an object is not to be considered unobservable merely because one is not in a position to observe it. concepts of space and time so that its meaninglessness is a necessary consequence. This Einstein did, and therein lies his greatness.

So fundamental is this principle that some of its requirements are at present far beyond the possibility of practical application : they belong to the future. Consider the age-old question : Does an object exist when no one is observing it? The first part of our principle immediately answers, No: for clearly it is impossible to observe an object at a moment when no one is observing it. Consequently, our final physical terms must be such that the question has no meaning.

Now the whole of what is known as 'field physics' necessarily involves existence without observation. Our observations are scattered, atomic, discontinuous, and we assume a continuum or continua (space, time, ether) in which they are distributed. Our field laws consequently describe realms of possibility rather than bits of actuality. The law of gravitation does not tell us the structure of the solar system. It gives us a prescription according to which an infinitude of solar systems might be built, but it cannot by its very nature tell us why we have our particular one. Our principle requires that this form of theory must be discarded.

Needless to say, the value of field theory as a means of advance is far from exhausted: it is its status as a possibly final form of scientific expression that is destroyed. Nor should this surprise us. A complete theory of the universethat is, of all that is physically observable-can scarcely be pictured as a set of super-universal laws supplemented by an independent statement of how, from some quite arbitrary starting-point, a particular system developed in accordance therewith. We should not be satisfied with any theory of the universe which did not give the details of the system equal inevitability with the laws according to which those details took shape. We can, indeed, deduce this directly from our principle. The universe cannot be regarded as one of a number of possible universes, because the others, being unobservable (our own comprises all that is observable), cannot exist. Hence our final account of it cannot be in terms of field theory.

From this point of view it is highly significant that during this same period of twenty-five years the other great branch of modern physics-quantum theory-has been transformed from the heretical speculation of a few daring theorists into a system with claims to universal scope. Quantum theory, unlike field theory, postulates no unrealised possibilities ; it opposes discontinuity to continuity, and seeks to describe the actual rather than the possible. It provides the very soil in which the 
principle of rejection of unobservables would be expected best to flourish.

The principle does flourish there, but again the result is generally seen more as a negative than as a positive achievement: we are far less conscious of the growing fruit than of the lost blossom. The supreme product of the quantum theory so far is described as a 'principle of uncertainty', and it is often regarded as having ousted causality from Nature. It is worth while looking at this matter for a moment in its historical setting.

The idea of causality in its elementary form is almost as old as thought itself : intelligent action is impossible without an assurance that a given act will be followed by an expected event. Only at a later stage of reflection is volition eliminated, so that the initial and final states of the physical system concerned are seen standing in causal connexion; and it is still later that the conception is extended throughout space, the state of the universe at one instant being regarded as the effect of its immediately preceding and the cause of its immediately succeeding state. This was the level of thought when Newton's laws of mechanics apparently placed the reality of the conception beyond question by discovering the clue to the inevitable succession of states. Newton gave formulæ by which, if the position and momentum of each particle of matter in the universe at any one instant were known, its position and momentum at the next instant could be determined, and hence its position and momentum throughout all time, supposing it to be eternal.

Newton's mechanics has been modified in various ways, and the study of radiation, electricity and such phenomena has revealed a richer physical universe than that which he contemplated, but none of these developments has destroyed the possibility of prescribing the data necessary to predict the future course of events. The fundamental modification of Newton's contribution to the idea of causality has come from the study of the means by which data are obtained. Minute investigation shows that it is impossible to determine exactly the simultaneous position and momentum of any particle of matter because our means of observation are such that precision in one determination can be obtained only at the expense of precision in the other.

It is important to see just what this means. In one sense it virtually puts us back to our position before Newton. We cannot state what are the data which would enable us to predict the future of the universe, but we may, as then, regard the predictability of its future as a generalisation of our experience of causality in limited systems of events. How far such generalisation is legitimate is indeed an important question, but it is not affected by recent work: Galileo could have discussed it with us without being at much disadvantage. Furthermore, the experience of causality in limited systems is a fundamental fact. It is impossible that scientific developments can overthrow it without destroying their legitimacy, for it is their basis. The sum and substance of the matter is that we have found that the data by which we thought we could forecast the future are unattainable.

There is an important difference between this and the statement which is frequently made, that the quantum theory requires that an experiment can be repeated several times under precisely similar conditions with various results. If that were true, it would indicate an irrationality in Nature which would be the negation of science. What the theory does show is that, if we define similarity of conditions as similarity of positions and momenta of the physical systems concerned, we can never be sure that we are repeating the experiment under precisely similar conditions. The distinction is profoundly important, for the actual situation leaves open the possibility that other data may be specified which will precisely identify a system, whereas the incorrect statement leaves no room for such a possibility.

Now it is just at this point that our fundamental principle of rejection of unobservables comes in. Since simultaneous position and momentum are unobservable, we must not only reject them, but we must also re-express our laws in terms according to which they have no meaning. Position and momentum are functions of the continua, time and space, which are appropriate to field theory, but cannot be expected precisely to fit phenomena which are essentially discontinuous. The problem of physics, then, is to devise other terms.

That such terms are possible there seems little reason to doubt. A useful beginning has been made with the concept of probability. This is expressed mathematically as a ratio of integers, and so is more appropriate to discrete phenomena than any kind of continuous extension. It is sometimes said that we can no longer use models to represent physical conceptions. This, of course, is inaccurate, or physics would have no place left for the man with imagination; it would be a sphere of action only for the robot. What has happened is that mechanical models, which are spatial, have given way to epistemological ones, which are integral. To regard this as anything but a step towards better conceptions is to miss the significance of the new enlightenment. The error of the nineteenth century physicists was not that they used mechanical models (which were 
entirely appropriate to their stage of development), but that they did not recognise them as models. To imagine that probability has any greater claim to inherent permanency than mechanisms-and, in particular, to draw fundamental conclusions from the accident that probability suggests an intrinsic uncertainty-is to make the same error.

It seems likely that the quantum theory, so far from expelling precision from our description of Nature, really opens the door to it for the first time. For strictly speaking, a field theory can never allow absolute precision since continua are infinitely divisible. A particle having a co-ordinate represented by a non-terminating decimal, for example, could have its position specified as nearly exactly as we pleased, but not with absolute exactness, and an infinite future would hold the possibility of an indefinite amount of departure from a prediction based on such specification. Data which must necessarily be expressed in integers, however, are clearly susceptible of absolutely exact expression. The present position is therefore that we have escaped from a scheme of thought which made precise prediction impossible into one which, though we are as yet less far advanced in it, offers absolute precision as a possible goal.

Comparisons, if not odious, are liable to be misleading, and it would be unwise to stress them. Nevertheless, it may well be doubted whether in any previous period of twenty-five years, physics has experienced a more substantial forward movement.

\section{Constitution of the Earth}

By Dr. Harold Jeffreys, F.R.S., St. John's College, Cambridge

COMPARING the position of geophysics now with what existed in 1910, while we are struck by the great development that has taken place, we are equally struck, on looking more closely, by the fact that most of the theoretical advances are due, not to specifically new methods, but to the fuller application of methods that were already known. The work of Kelvin and Sir George Darwin on the rigidity of the earth, and on the evolution of the earth-moon system under the action of tidal friction, was already classical; Darwin's theory of the stresses needed to support continents and mountains was thirty years old; the existence of isostatic compensation, and the two alternative explanations of Pratt and Airy, had been known for fifty years, Stokes's theory of the determination of the figure of the earth from observations of gravity for sixty, and Poisson's theory of the longitudinal and transverse waves in an elastic solid for eighty. Dr. C. Davison, still with us, had put the thermal contraction theory of mountain formation on a quantitative basis in 1887, and Wiechert had shown how to reconcile the earth's ellipticity and precessional constant on the assumption of a thick rocky shell surrounding a dense metallic core. The existence of a change in properties in the crust in the continents at some small depth had already been inferred from geological considerations by Suess.

The chief new advance in the first ten years of the present century probably arose from the detection of radioactivity, the recognition of its effect in modifying the earth's thermal history, and the use of the rate of disintegration of uranium to find the absolute ages of minerals and to calibrate the geological time-scale. The age of the earth, estimated from thermal considerations by Kelvin at about 20 million years, was suddenly raised to about 1,500 million. Physicists did not all accept the new estimate without a struggle, though purely mechanical considerations might have given some ground for doubting Kelvin's value. Darwin, by adopting such a viscosity in the earth as would make the changes through tidal friction occur at every time at the maximum rate, the viscosity thus varying with time in a way very unlikely to correspond to the facts, could not bring the age of the moon below 54 million years. This might have been taken as an absolute minimum that was practically certain to be greatly exceeded.

The new source of heat was so potent that the present Lord Rayleigh pointed out that, if it was not confined to a depth of some tens of kilometres at the outside, it would produce more heat than is escaping from the earth; consequently it led Holmes to estimate the rate of decrease with depth. It was found to suggest that average granite could exist only to a depth of about $15 \mathrm{~km}$. and agreed in principle with the conclusions of Suess.

Meanwhile, seismology made three great advances. Herglotz and Bateman provided a method of finding the velocity of an elastic wave at any depth in the earth from the observed times of travel of earthquake waves, which was first applied by S. Mohorovičić in 1916. R. D. Oldham found that longitudinal waves arrived at the 